\title{
A normal electrocardiogram precludes the need for left ventriculography in the assessment of coronary artery disease
}

\author{
M A Khan, S Sinha, S Hayton, S Fynn, R A Henderson, D H Bennett
}

\begin{abstract}
Objective-To assess whether a normal electrocardiogram can identify good left ventricular function and obviate the need for routine left ventriculography in patients undergoing cardiac catheterisation for suspected coronary artery disease.

Design-A prospective study of patients undergoing cardiac catheterisation. Setting-A regional cardiac centre.

Patients-The electrocardiograms, coronary angiograms, and left ventriculograms of 391 consecutive patients undergoing investigations for suspected coronary artery disease were entered into the study. Patients with arrhythmias and cardiac pathologies other than coronary artery disease were excluded.
\end{abstract}

Main outcome measures-The electrocardiogram was assessed using a 29 point QRS scoring system, and classified by two cardiologists and a trainee cardiologist as normal or abnormal. Left ventricular function was assessed by digital ventriculography.

Results-The sensitivity, specificity, and negative predictive value of a QRS score of 0 (normal QRS complexes) for discriminating good left ventricular function (ejection fraction $\geqslant 50 \%$ ) were $92.6 \%, 41.5 \%$, and $97.2 \%$, respectively. The figures for a normal electrocardiogram as assessed by a doctor were $96.3 \%, 40.4 \%$, and $98.6 \%$ for cardiologist $A ; 96.3 \%, 37.4 \%$, and $98.4 \%$ for cardiologist $B$; and $94.4 \%, 49.6 \%$, and $\mathbf{9 8 . 2 \%}$ for the cardiology trainee.

Conclusions-If a cardiologist judges the ECG to be normal, left ventriculography is unnecessary and a formal QRS score does not improve reliability of this clinical judgment. Adopting this strategy would save $£ 30-40000$ in consumables and $65-87$ hours of catheter laboratory and staff time for a department catheterising 3000 patients with suspected coronary artery disease annually.

(Heart 1998;79:262-267)

Keywords: cardiac catheterisation; electrocardiogram; left ventricular ejection fraction

Left ventricular function is an important predictor of long term outcome in patients with coronary artery disease and may influence clinical management. Patients with impaired ventricular function have been shown to have improved outcome on angiotensin converting enzyme (ACE) inhibitor treatment. ${ }^{1-3}$ If they have three vessel disease or left main stem disease, coronary artery bypass grafting (CABG) improves survival. ${ }^{4}$ Surgical risks may be increased in patients with severe left ventricular dysfunction. Thus left ventriculography is usually performed routinely in patients undergoing coronary angiography for suspected coronary artery disease.

Left ventricular function can also be assessed non-invasively from the electrocardiogram (ECG). A 32 point QRS scoring system to assess left ventricular function has been described. ${ }^{5}$ It showed a high negative correlation between the QRS score and left ventricular function ( $\mathrm{n}=55, r=-0.88)$ in patients with recent myocardial infarction. Further studies have supported the negative relation between ECG scores and left ventricular function, ${ }^{6-9}$ while Fioretti et al also used a QRS score to identify patients at low or high risk of death following myocardial infarction. ${ }^{10}$ Subsequently, Selvester's 32 point QRS score was simplified to a 29 point score, which was easier to use and had a high inter- and intraobserver reproducibility. ${ }^{11}$ However, later studies have questioned the strength of the relation between QRS score and left ventricular function, particularly in patients with non-recent myocardial infarction, and concluded that the QRS score has a high variability and cannot predict left ventricular function reliably in individual patients..$^{91013}$ All these studies have used non-invasive methods of assessing left ventricular function, while none has specifically focused on patients undergoing cardiac catheterisation for the investigation of coronary artery disease. This is a heterogeneous group consisting of patients with both recent and non-recent myocardial infarction, patients with no previous history of infarction, and patients who have undergone revascularisation; thus it is difficult to draw conclusions about this group from published studies.

We therefore prospectively assessed whether ECG evaluation in patients undergoing cardiac catheterisation for suspected coronary artery disease can reliably identify those with good left ventricular function, in whom left ventriculography may be unnecessary.

\section{Methods}

Consecutive patients undergoing cardiac catheterisation for suspected coronary artery disease without arrhythmias, valvar heart disease, other cardiac pathology were entered into the study. 
Table 1 QRS scoring system

\begin{tabular}{|c|c|c|c|c|c|}
\hline Lead & Duration (ms) & Points & Amplitude ratios & Points & $\begin{array}{l}\text { Maximum } \\
\text { score }\end{array}$ \\
\hline I & $Q \geqslant 30$ & 1 & $\mathrm{R} / \mathrm{Q} \leqslant 1$ & 1 & 2 \\
\hline \multirow[t]{2}{*}{ II } & $\mathrm{Q} \geqslant 40$ & 2 & & & \\
\hline & $\mathrm{Q} \geqslant 30$ & 1 & & & 2 \\
\hline aVL & $Q \geqslant 30$ & 1 & $\mathrm{R} / \mathrm{Q} \leqslant 1$ & 1 & 2 \\
\hline \multirow[t]{3}{*}{$\mathrm{aVF}$} & $Q \geqslant 50$ & 3 & $\mathrm{R} / \mathrm{Q} \leqslant 1$ & 2 & \\
\hline & $Q \geqslant 40$ & 2 & & & \\
\hline & $Q \geqslant 30$ & 1 & $\mathrm{R} / \mathrm{Q} \leqslant 2$ & 1 & 5 \\
\hline \multirow[t]{3}{*}{ V1 } & Any $Q$ & 1 & & & \\
\hline & $R \geqslant 50$ & 2 & & & \\
\hline & $R \geqslant 40$ & 1 & $\mathrm{R} / \mathrm{S} \geqslant 1$ & 1 & 4 \\
\hline \multirow[t]{3}{*}{$\mathrm{V} 2$} & Any $Q$ or $R \leqslant 20$ & 1 & & & \\
\hline & $R \geqslant 60$ & 2 & & & \\
\hline & $R \geqslant 50$ & 1 & $\mathrm{R} / \mathrm{S} \geqslant 1.5$ & 1 & 4 \\
\hline V3 & Any $Q$ or $R \leqslant 30$ & 1 & & & 1 \\
\hline \multirow[t]{2}{*}{ V4 } & $Q \geqslant 20$ & 1 & $\mathrm{R} / \mathrm{Q}$ or $\mathrm{R} / \mathrm{S} \leqslant 0.5$ & 2 & \\
\hline & & & $\mathrm{R} / \mathrm{Q}$ or $\mathrm{R} / \mathrm{S} \leqslant 1$ & 1 & 3 \\
\hline \multirow[t]{2}{*}{ V5 } & $Q \geqslant 30$ & 1 & $\mathrm{R} / \mathrm{Q}$ or $\mathrm{R} / \mathrm{S} \leqslant 1$ & 2 & \\
\hline & & & $\mathrm{R} / \mathrm{Q}$ or $\mathrm{R} / \mathrm{S} \leqslant 2$ & 1 & 3 \\
\hline \multirow[t]{2}{*}{ V6 } & $Q \geqslant 30$ & 1 & $\mathrm{R} / \mathrm{Q}$ or $\mathrm{R} / \mathrm{S} \leqslant 1$ & 2 & \\
\hline & & & $\mathrm{R} / \mathrm{Q}$ or $\mathrm{R} / \mathrm{S} \leqslant 3$ & 1 & 3 \\
\hline
\end{tabular}

Reproduced with permission from Circulation 1982;65:342-7.

Table 2 Study patient risk factors

\begin{tabular}{llr}
\hline Characteristic & \multicolumn{1}{l}{ Present } & \multicolumn{1}{c}{ Unknown $^{\star}$} \\
\hline Hypertension & $32.7 \%(128 / 391)$ & $4.6 \%(18 / 391)$ \\
Hypercholesterolaemia & $60.1 \%(235 / 391)$ & $18.7 \%(73 / 391)$ \\
Myocardial infarction & $45.3 \%(177 / 391)$ & $4.6 \%(18 / 391)$ \\
Diabetes & $10.0 \%(39 / 391)$ & $4.3 \%(17 / 391)$ \\
Smoking & & \\
$\quad$ Current & $6.1 \%(24 / 391)$ & \\
Ex-smoker & $66.2 \%(259 / 391)$ & \\
Never smoked & $21.7 \%(85 / 391)$ &
\end{tabular}

Hypercholesterolaemia, fasting cholesterol $\geqslant 5.2 \mathrm{mmol} / \mathrm{l}$. *Presence or absence of diagnosis not confirmed by patient or case notes.

ELECTROCARDIOGRAM

A standard 12 lead ECG was performed on all patients immediately before cardiac catheterisation. The ECGs were analysed without knowledge of the patient's details, using a modified QRS score (table 1), ${ }^{11}$ which involved a weighted scoring system using $Q$ and $R$ wave duration and $\mathrm{R} / \mathrm{Q}$ and $\mathrm{R} / \mathrm{S}$ amplitude ratios. The range of possible scores is 0 to 29, 0 representing a normal QRS pattern. ECGs showing left ventricular hypertrophy, ${ }^{14}$ bundle branch block, or axis deviation (frontal plane axis $\leqslant 45^{\circ}$ and $\geqslant 100^{\circ}$ ) were excluded by Wagner et $a l^{11}$ and were not validated with the QRS scoring system we have used. Therefore, ECGs with these abnormalities were considered to have a QRS score greater than 0 .

The ECG was also assessed by two experienced cardiologists and a cardiology trainee unaware of patient details, and classified as normal or abnormal. For this aspect of the ECG assessment, no criteria for normality

Table 3 Severity of coronary artery disease and presence of previously undiagnosed mitral regurgitation

\begin{tabular}{|c|c|c|c|c|c|c|c|}
\hline \multirow{2}{*}{$\begin{array}{l}\text { Number of vessels } \\
\text { with } \geqslant 50 \% \\
\text { stenosis }\end{array}$} & \multicolumn{5}{|c|}{ Number (\%) of vessels with reduced TIMI flow } & \multicolumn{2}{|c|}{ Mitral regurgitation } \\
\hline & 0 & 1 & 2 & 3 & Total & Moderate & Severe \\
\hline 0 & $71(18)$ & 0 & 0 & 0 & $71(18)$ & 0 & 0 \\
\hline 1 & $34(9)$ & $27(7)$ & 0 & 0 & $61(16)$ & 0 & 0 \\
\hline 2 & $35(9)$ & $40(10)$ & $18(5)$ & 0 & $93(24)$ & 0 & 0 \\
\hline 3 & $35(9)$ & $80(21)$ & $42(11)$ & $8(2)$ & $165(42)$ & 4 & 0 \\
\hline
\end{tabular}

TIMI, blood flow as defined by the thrombolysis in myocardial infarction trial, reduced blood flow $=$ grade 2 or less.

${ }^{\star}$ Details of coronary anatomy, blood flow, and mitral regurgitation not available for one patient (patient had an abnormal ECG and QRS score). were prespecified. The observers were asked to classify the QRS complex and ST/T waves as "normal" or "abnormal," and to apply the definitions that they personally used in routine clinical practice. The only recommendation made was that a truly borderline abnormality was placed in the "abnormal" category.

\section{CARDIAC CATHETERISATION}

Patients underwent coronary angiography and left ventriculography using an International General Electric single plane LC omega IIIA with Advantx DX-C Hilina digital cardiac imaging system (IGE Medical Systems, Slough, Bucks, UK). Left ventriculography was performed in the right anterior oblique view and reviewed without knowledge of the patient's details. Images with inadequate delineation of endocardial contours at end diastole or end systole, or images taken during a non-sinus or postectopic beat were excluded. Global ejection fraction and end systolic volume were automatically calculated using Sadler and Dodge's method and corrected for body surface area. ${ }^{15}$ Abnormal left ventricular function was defined as an ejection fraction $<50 \%$, while an abnormal end systolic volume was defined as $>50 \mathrm{ml} / \mathrm{m}^{2}$. The presence of mitral regurgitation was assessed visually and classified as none, trivial, mild, moderate, or severe. Regional wall motion was estimated using the centreline method, which measures movement along 100 cords drawn perpendicular to a centreline constructed midway between the end diastolic and end systolic contours. ${ }^{16}$ The motion is normalised for heart size by dividing by the length of the end diastolic perimeter. Motion at each cord was expressed in units of standard deviation (SD) from a normal population mean. Significant cord hypokinesis and hyperkinesis were defined as wall motion $2 \mathrm{SD}$ above or below the normal population mean, respectively. Significant left ventricular hypokinesis and hyperkinesis were defined arbitrarily as wall motion abnormality affecting more than 16 of 80 cords $(20 \%)$, excluding cords representing the mitral valve. Diastolic function was not assessed.

The coronary angiograms were also reviewed without knowledge of the patient's details. Significant coronary artery stenosis was defined as a reduction of at least $50 \%$ in the diameter of a major coronary artery or important branch. The numbers of vessels affected and the blood flow, as defined by the thrombolysis in myocardial infarction trial (TIMI), were recorded. ${ }^{17}$

\section{STATISTICS}

Standard statistics were used to describe the patients' characteristics. We analysed the sensitivity, specificity, and predictive values of the ECG (using both cardiologist assessed and QRS score methods) in predicting abnormal left ventricular function (ejection fraction $<50 \%$, end systolic volume $>50 \mathrm{ml} / \mathrm{m}^{2}$, or more than 16 hypokinetic cords). Abnormal ECG and abnormal left ventricular function were deemed to be positive results. These values are defined as follows. Sensitivity (true 
Table 4 Patient distribution according to ECG and left ventricular function

\begin{tabular}{|c|c|c|c|c|c|c|}
\hline & $E F \geqslant 50 \%$ & $E F<50 \%$ & $\begin{array}{l}\text { Sensitivity } \\
(\%)\end{array}$ & $\begin{array}{l}\text { Specificity } \\
(\%)\end{array}$ & $\begin{array}{l}N P V \\
(\%)\end{array}$ & $\begin{array}{l}\text { Cost savings } \\
(£)\end{array}$ \\
\hline \multicolumn{7}{|c|}{ Cardiologist $A$} \\
\hline Normal & 136 & 2 & 96.3 & 40.4 & 98.6 & 33565 \\
\hline Abnormal & 201 & 52 & & & & \\
\hline \multicolumn{7}{|c|}{ Cardiologist $B$} \\
\hline Normal & 126 & 2 & 96.3 & 37.4 & 98.4 & 31132 \\
\hline Abnormal & 211 & 52 & & & & \\
\hline \multicolumn{7}{|l|}{ Trainee } \\
\hline Normal & 167 & 3 & 94.4 & 49.6 & 98.2 & 41348 \\
\hline Abnormal & 170 & 51 & & & & \\
\hline \multicolumn{7}{|l|}{ QRS score ${ }^{\star}$} \\
\hline 0 & 140 & 4 & 92.6 & 41.5 & 97.2 & 35024 \\
\hline$\geqslant 1$ & 197 & 50 & & & & \\
\hline
\end{tabular}

^29 point QRS score.

$\mathrm{EF}$, ejection fraction; NPV, negative predictive value.

positive $)=$ the number of patients correctly identified as having abnormal left ventricular function / total number of patients with abnormal left ventricular function. Specificity (true negative $)=$ the number of patients correctly identified as having preserved left ventricular function / total number of patients with preserved left ventricular function. Negative predictive value (utility of a negative result $)=$ the number of patients correctly identified as having preserved left ventricular function / total number of patients with a normal ECG. Positive predictive value (utility of a positive result $)=$ the number of patients correctly identified as having abnormal left ventricular function / total number of patients with an abnormal ECG.

The relations between the normal QRS score and ejection fraction and end systolic volume corrected for body surface area were also assessed using Pearson's correlation coefficient. The interobserver agreement for cardiologist/trainee ECG assessment was measured by calculating the percentage of ECGs that all three observers agreed upon. Simple arithmetical analysis was performed to assess possible cost savings, if a policy of omit-

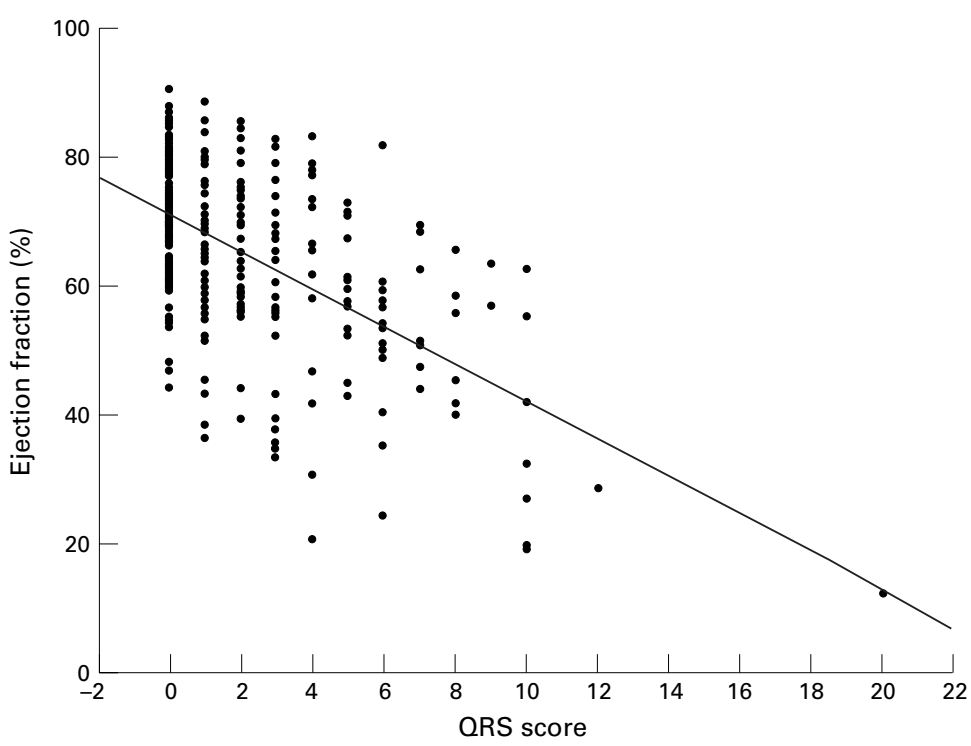

Figure 1 A comparison between ejection fraction and $Q R S$ score.

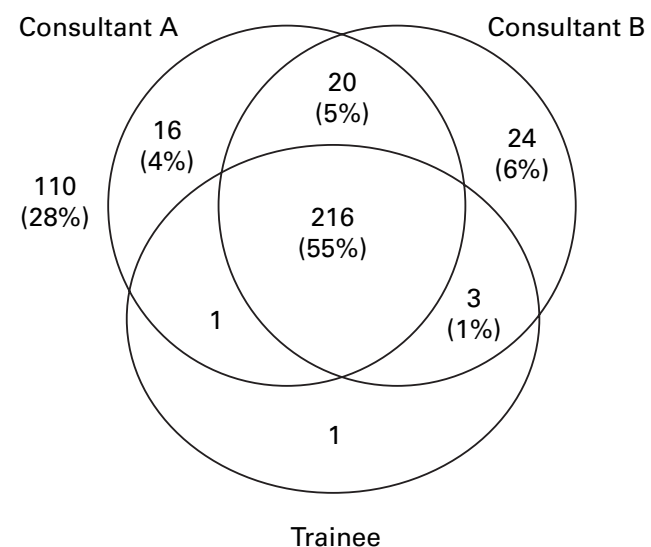

Figure 2 A Venn diagram to show differences in ECG interpretation between the three doctors. Numbers inside circles represent ECGs classified as abnormal.

ting left ventriculography in patients with a normal ECG was adopted.

\section{Results}

Three hundred and ninety one patients were entered into the study-305 males and 86 females, mean age 56.9 years (range 27 to 76 years). Angina and breathlessness were present in $88 \%$ and $52 \%$ of the patients, respectively ( $\mathrm{n}=344$ and 202). Breathlessness alone was present in only eight patients $(2 \%)$. Patient risk factors are shown in table 2 . The severity of coronary artery disease detected at angiography and the presence of moderate/severe mitral regurgitation is shown in table 3 .

\section{ECG SCORE}

Patients with abnormal QRS scores tended to have a lower ejection fraction than those with normal QRS scores (fig 1) although the correlation coefficient was low $(r=-0.58$, $\mathrm{p}<0.001)$. There was a similar small negative correlation between end systolic volume and QRS score, $r=-0.49, \mathrm{p}<0.001$.

Despite the low correlation between ejection fraction and QRS score, of 144 patients with a QRS score of 0 only four had abnormal left ventricular function with an ejection fraction of $44 \%$ to $49 \%$. No patients with a QRS score of 0 had moderate or severe mitral regurgitation. In addition, only two patients had a mildly raised end systolic volume (50.5 to 52.1 $\mathrm{ml} / \mathrm{m}^{2}$ ). The specificity, sensitivity, and negative predictive values are shown in table 4 .

Overall, there were 32 patients with significant segmental wall abnormalities. Only one patient had a normal QRS score and would not have undergone left ventriculography if our suggested policy had been implemented.

\section{CARDIOLOGIST ASSESSED ECG}

There was good agreement in ECG interpretation between any two of the three observers: $88 \%(347 / 391)$ to $90 \%$ (351/391), with $83 \%$ agreement (326/391) between all three observers (fig 2).

The selection of normal ECGs by the two experienced cardiologists to predict left ventricular function resulted in only two false negatives, where abnormal left ventricular 
function was missed (ejection fraction $44 \%$ to $48 \%$, end systolic volume 40 to $49.5 \mathrm{ml} / \mathrm{m}^{2}$ ). Similarly, the trainee assessment of normal ECGs to predict left ventricular function resulted in only three false negatives (ejection fraction $44 \%$ to $48 \%$ ). The specificity, sensitivity, and negative predictive values for all three observers are shown in table 4. No patients with a normal ECG had had moderate or severe mitral regurgitation. In addition, one patient had a mildly raised end systolic volume $\left(52.1 \mathrm{ml} / \mathrm{m}^{2}\right)$.

Overall, there were 32 patients with significant segmental wall motion abnormalities. Only one patient had a normal ECG and would not have undergone left ventriculography if our suggested policy had been implemented.

COST SAVINGS

Assuming a saving of $40 \mathrm{ml}$ of contrast $(£ 20)$ and one angiographic catheter $(£ 11.70)$ per case, a department catheterising 3000 patients annually for suspected coronary artery disease would save between $£ 30000$ and $£ 40000$ in consumables if patients with a QRS score of 0 or a normal ECG are spared routine ventriculography. Potential cost savings using each individual cardiologist's assessment or the QRS score are detailed in table 4. In addition, there would be a potential time saving of between 65 and 87 hours, assuming an average of four minutes (our own unpublished observations) to perform left ventriculography.

\section{Discussion}

Our results support the hypothesis that patients with either a normal ECG or a QRS score of 0 who are undergoing cardiac catheterisation for suspected coronary artery disease do not require routine left ventriculography. There was substantial agreement between the ECG assessments of all three observers (fig 2) and in keeping with previous studies, ECG interpretation was not significantly different between the consultants and the registrar grade trainee. ${ }^{18}$ Sensitivities, specificities, and negative predictive values were very similar to those obtained using a more formal QRS scoring system (table 4). In day to day practice, QRS scoring would be a cumbersome procedure and we advocate the use of a physician assessed ECG in preference.

The original studies using the QRS scoring system suggested that there was a close linear relation between the QRS score and left ventricular function (ejection fraction $=60-3$ $\times$ QRS score) with a high correlation, which may be of use in calculating ejection fraction from the ECG. ${ }^{5}$ Although we also showed a negative correlation between QRS score and the ejection fraction, the strength of this correlation was low $(r=-0.58)$ reflecting a marked variability in ejection fraction at any given QRS score. Our data are in agreement with those from several other groups who report low correlation coefficients, and have questioned the value of the QRS score in predicting the ejection fraction in individual patients. ${ }^{9} 101213$ Previous studies have mostly been limited by relatively small numbers of patients and have only investigated those with definite previous myocardial infarction. In this study we investigated a larger and more heterogeneous group of patients, including those with recent, non-recent, and no prior myocardial infarction, which may account for the high variability in ejection fraction observed. We have also extended our analysis to investigate specifically the value of a normal ECG or a QRS score of 0 . Although the variability in ejection fraction remains high in patients with a normal ECG, we showed that none of them had a clinically significant degree of left ventricular dysfunction.

Our aim was to determine whether the ECG could be used to identify patients who do not need left ventriculography during catheterisation for coronary artery disease. We evaluated a cardiologist assessed ECG, but in view of the potential variation in ECG interpretation between clinicians, we also used a formal 29 point QRS score. ${ }^{11}$ Selvester's original 32 point score includes qualitative criteria, such as slurs or notches, that are difficult to apply uniformly to a large number of ECGs by different observers, as well as criteria involving the absolute amplitude of a deflection which is highly variable in the normal population. We therefore elected to use the simplified 29 point version of Selvester's original QRS score, which omits the above criteria and has been shown to have a high inter- and intraobserver reproducibility. ${ }^{11}$

We adopted a conservative strategy with the aim of identifying all patients with significant left ventricular dysfunction (as failure to identify this subgroup could have serious clinical implications), with ECG criteria that resulted in the highest possible sensitivity for predicting abnormal ventricular function. Accordingly, we used the criteria of normal ECG or QRS score of 0 to identify patients with normal left ventricular function. Our results indicate that a normal ECG or QRS score of 0 has high negative predictive values ( $97 \%$ to $99 \%$ ) and only between two and four false negatives, regardless of the ECG assessment technique or the observer. Moreover, all of these had only a mild degree of left ventricular dysfunction that was of little clinical significance. However, this strategy would lead to a loss of specificity, resulting in many patients with mild or borderline ECG abnormalities and normal ventricular function undergoing ventriculography, thus negating some potential cost savings.

It would be possible to vary these ECG criteria and to plot a receiver-operator curve (ROC), hence documenting a range of sensitivities and specificities for the different ECG criteria used. This approach has been followed in some studies that have used the ECG to predict infarct size, as assessed non-invasively, and allows investigators the opportunity to decide for themselves whether they would favour criteria with high sensitivity and low specificity or vice versa. ${ }^{10}$ However, reanalysis of the QRS score and ejection fraction data, with planned omission of ventriculography in patients with a QRS score of 0 or 1 , resulted in twice as many false negatives $(n=8)$, where abnormal left ventricular function was missed (ejection frac- 
tion $36 \%$ to $48 \%$, end systolic volume 26 to $77 \mathrm{ml} / \mathrm{m}^{2}$, data not shown). Of these patients, two had ejection fractions of less than $40 \%$, a degree of left ventricular dysfunction that may have implications for their future management. We would therefore recommend a conservative approach to the ECG criteria used (maintaining the highest possible sensitivity), as we feel the priority is to ensure that even a few patients with significant left ventricular dysfunction do not fail to have ventriculography, and a ROC would not be helpful in this context.

It is possible that normal global ejection fraction and end systolic volume may coexist with significant regional wall motion abnormalities. Consequently, we assessed wall motion abnormalities using the centreline method, and found that only one patient with a normal ECG or a QRS score of 0 had significant wall motion abnormalities (with 26/80 hypokinetic cords). However, as this patient also had a normal ejection fraction and end systolic volume, the prognostic and clinical significance of wall motion abnormality with otherwise normal left ventricular function is uncertain. All other patients with significant regional wall motion abnormalities also had an abnormal ECG, and in keeping with our suggested management strategy would require ventriculography. Furthermore, it is likely that we have overestimated the number of patients with regional wall motion abnormality since a major limitation of all angiographic methods of assessing ventricular function-including the centre line method adopted in this study-is the difficulty in distinguishing regional wall motion abnormalities from translational movements of the heart or asynchronous contraction.

A potential drawback of the omission of routine ventriculography in all patients is the loss of information concerning undiagnosed mitral regurgitation and left ventricular pressures which may be important in the assessment of diastolic left ventricular dysfunction. We assessed the presence of mitral regurgitation on left ventriculography and found that no patient with a QRS score of 0 or a normal cardiologist assessed ECG had moderate or severe mitral regurgitation. Regarding diastolic left ventricular dysfunction there is still debate as to the best method of measurement, the best prognostic indicator, and the optimum treatment. ${ }^{19}$ The natural history is poorly characterised but the prognosis appears to be substantially better than in patients with systolic left ventricular dysfunction. ${ }^{20}$ In view of these concerns, we did not feel that the benefits of obtaining left ventricular pressure information from all patients were sufficient to outweigh the cost and time savings acquired by omitting ventriculography in selected patients. Clearly, if mitral regurgitation or diastolic dysfunction are suspected as principal diagnoses, then ventriculography may be warranted in association with other investigations, even in the presence of a normal ECG.

There may be a perceived difficulty in applying the results of this study to clinical practice in particular subgroups. In the case of women alone, similar results were obtained to those presented for the whole group (data not shown). There was also concern that our findings may not apply to patients with severe coronary lesions that limit anterograde flow or blockages with retrograde flow. Table 3 shows the number of patients with reduced TIMI flow or retrograde filling in this study. A significant number of such patients were represented (with 55\% having reduced TIMI flow in at least one vessel) and the predictive values, sensitivity, and specificity of a normal ECG or a QRS score of 0 were similar to those in the whole study (results of subgroup analysis not shown). We therefore feel that our conclusions are generally valid, although the smaller numbers involved in subgroup analysis will inevitably reduce confidence in our conclusions regarding any particular subgroup.

Another potential limitation of this study is the fact that patients on $\beta$ blocking agents were not advised to stop their treatment before cardiac catheterisation, and theoretically this may depress cardiac function with no concomitant ECG change. However, the effects of oral $\beta$ blockade on resting left ventricular function have been well characterised, with most studies reporting no change or an increase in ejection fraction, while a few report small but clinically insignificant reductions in ejection fraction. ${ }^{21}$ More recently, controlled trials of various $\beta$ blockers show that these drugs can increase left ventricular function even in patients with heart failure. ${ }^{22}{ }^{23}$ Our unit policy is therefore to continue $\beta$ blockade during cardiac catheterisation and we do not believe this confounds the assessment of left ventricular function significantly.

In summary, we have shown that a normal ECG or a QRS score of 0 can obviate the need for left ventriculography in patients with suspected coronary artery disease without loss of important clinical data, resulting in a saving of $£ 30000$ to $£ 40000$ in consumables and 65 to 87 hours of catheter laboratory and staff time, for a department catheterising 3000 patients annually with suspected coronary artery disease. Further benefits would include lower radiation exposure to patients and staff, and prolongation of the life of the cathode ray tube. This policy is most conveniently effected by using physician assessed ECGs, which compare favourably with a formal QRS scoring system and show a high level of reproducibility. Since performing this study, we have successfully implemented this policy in our unit and would recommend it to other cardiology departments.

We thank the ECG and cardiac radiology departments, Barbara Pickering, and Mary Rooney for their help and cooperation with this study. The study was funded by Wythenshawe Hospital Cardiology Research Fund, registered charity number 801760.

1 The CONSENSUS Trial Study Group. Effects of enalapril on mortality in severe congestive heart failure. Results of the Cooperative North Scandinavian Enalapril Survival Study (CONSENSUS). N Engl f Med 1987;316:1429-35. 2 SOLVD Investigators. Effects of enalapril on survival in patients with reduced left ventricular ejection fraction and patients with reduced left ventricular ejection fraction and congestive heart failure. $N$ Engl f Med 1991;325:293-302. Pfeffer MA, Braunwald E, Moyye LA, et al. Effects of captopril on mortality and morbidity in patients with left ventricular dysfunction after myocardial infarction. Results of the Survival and Ventricular Enlargement Trial (SAVE). 
4 CASS principle investigators and their associates. Myocardial infarction and mortality in coronary artery surgery 5tudy

5 Palmeri ST, Harrison DG, Cobb FR, et al. Evaluation of a QRS scoring system for assessing left ventricular function after myocardial infarction. N Engl f Med 1982;306:4-9.

6 Anderson CI, Harrison DG, Stack NC, et al. Evaluation of serial QRS changes during acute inferior myocardial using a QRS scoring system. Am f Cardiol 1983;52:252-6.

7 Ideker RE, Wagner GS, Ruth WK, et al. Evaluation of a QRS scoring system for estimating myocardial infarction
size. II. Correlation with quantitative anatomic findings for anterior infarcts. Am $\mathcal{F}$ Cardiol 1982;49:1604-14

8 Roark SF, Ideker RE, Wagner GS, et al. Evaluation of a QRS scoring system for estimating myocardial infarction size. III. Correlation with quantitative anatomic findings for inferior infarcts. Am 7 Cardiol 1983;52:382-9.

9 De Pace NL, Abdulmassih S, Iskandrian AS, et al. Use of QRS scoring and thalium-201 scintigraphy to assess left ventricular function after myocardial infarction. Am 7 Carventricular function
diol $1982 ; 50: 1262-8$

10 Fioretti P, Brower RW, Lazzeroni E, et al. Limitation of QRS scoring system to assess left ventricular function and prog nosis at hospital discharge after myocardial infarction. $B$ Heart f 1985;53:248-52

11 Wagner GS, Freye CJ, Palmeri ST, et al. Evaluation of a QRS scoring system for estimating myocardial infarction size. I. Specificity and observer agreement. Circulation 1982;65:342-7.

12 Young SG, Abouantoun S, Savvides M, et al. Limitations of electrocardiographic scoring system for estimation of left ventricular function. Am 7 Cardiol 1983;52:1479-88.

13 Seinio Y, Staniloff HM, Shell WE, et al. Evaluation of a QRS scoring system in acute myocardial infarction: relation to infarct size, early stage left ventricular ejection fraction, and exercise performance. Am f Cardiol 1983;52:37-42.
14 Rowlands DJ. The resting electrocardiogram. In: Julian DG, ed. Diseases of the heart. London: Bailliere Tindall, 1989:161.

15 Sadler H, Dodge HT. The use of single plane angiocardiorams for the calculation of left ventricular volume in man. Am Heart F 1968;75:325-34.

16 Sheehan FH, Bolson EL, Dodge HT, et al. Advantages and application of the centerline method for characterizing regional ventricular function. Circulation 1986;74:293-305.

17 Chesebro JH, Knatterud G, Roberts R, et al. Thrombolysis in myocardial infarction (TIMI) trial, phase I: a comparison between intravenous tissue plasminogen activator and intravenous steptokinase. Circulation 1987;76:142-54.

18 Khan MA, King D, Davies KN, et al. Practical cardiological skills and ECG interpretation amongst doctors. $\mathrm{Br} F \mathrm{Clin}$ Pract 1993;47:183-4.

19 Vasan RS, Benjamin EJ, Levy D. Congestive heart failure with normal left ventricular systolic function. Clinical approaches to the diagnosis and treatment of diastolic heart failure. Arch Intern Med 1996;156:146-57.

20 Gaasch WH. Diagnosis and treatment of heart failure based on left ventricular systolic or diastolic dysfunction. $尹 A M A$ 1994;271:1276-80.

21 Nestico PF, Hakki AH, Iskandrian AS. Effects of cardiac medication on ventricular performance: emphasis on evaluation with radionuclide angiography. Am Heart $\mathcal{f}$ 1985;109:1070-84.

22 Packer M. Beta-blockade in the management of chronic heart failure. Another step in the conceptual evolution of a neurohormonal model of the disease. Eur Heart $f$ 1996;17(suppl B):21-3.

23 Fisher ML, Gottlieb SS, Plotnick GD, et al. Beneficial effects of metoprolol in heart failure associated with coronary artery disease: a randomised trial. $\mathcal{F} \mathrm{Am}$ Coll Cardiol 1994;23:943-50. 